\title{
Association between concurrent use of prescription opioids and benzodiazepines and overdose: retrospective analysis
}

\author{
Eric C Sun, ${ }^{1}$ Anjali Dixit, ${ }^{2}$ Keith Humphreys, ${ }^{3}$ Beth D Darnall, ${ }^{1}$ Laurence C Baker, ${ }^{4}$ Sean Mackey ${ }^{1}$
}

'Department of Anesthesiology, Perioperative and Pain

Medicine, Stanford University

School of Medicine, 300

Pasteur Dr, H3580, Stanford, CA

94305, USA

${ }^{2}$ Department of Anesthesiology and Perioperative Care,

University of California, San

Francisco, 521 Parnassus Ave,

San Francisco, CA 94131, USA

${ }^{3}$ Center for Innovation to Implementation, VA Palo Alto

Health Care System and

Department of Psychiatry,

Stanford University School of

Medicine, Stanford University,

401 N Quarry Road, MC:5717,

Stanford, CA 94305, USA

${ }^{4}$ Department of Health Research and Policy, Stanford University

School of Medicine, Stanford

University and National Bureau

of Economic Research, 150

Governor's Lane, HRP Redwood

Building, Stanford, CA 94305,

USA

Correspondence to: ESun

esun1@stanford.edu

Additional material is published online only. To view please visit the journal online.

Cite this as: $B M J$ J 2017;356:j760 http://dx.doi.org/10.1136/bmj.j760

Accepted: 30 January 2017

\section{ABSTRACT}

OBJECTIVES

To identify trends in concurrent use of a

benzodiazepine and an opioid and to identify the

impact of these trends on admissions to hospital and emergency room visits for opioid overdose.

DESIGN

Retrospective analysis of claims data, 2001-13.

SETTING

Administrative health claims database.

PARTICIPANTS

315428 privately insured people aged 18-64 who were continuously enrolled in a health plan with medical and pharmacy benefits during the study period and who also filled at least one prescription for an opioid.

\section{INTERVENTIONS}

Concurrent benzodiazepine/opioid use, defined as an overlap of at least one day in the time periods covered by prescriptions for each drug.

\section{MAIN OUTCOME MEASURES}

Annual percentage of opioid users with concurrent benzodiazepine use; annual incidence of visits to emergency room and inpatient admissions for opioid overdose.

\section{RESULTS}

$9 \%$ of opioid users also used a benzodiazepine in 2001 , increasing to $17 \%$ in 2013 (80\% relative increase). This increase was driven mainly by increases among intermittent, as opposed to chronic, opioid users. Compared with opioid users who did not use benzodiazepines, concurrent use of both drugs was associated with an increased risk of an emergency

\section{WHAT IS ALREADY KNOWN ON THIS TOPIC}

In many countries, the increased use of prescription opioids and the resulting potential for addiction and overdose represent a growing public health concern Nearly $30 \%$ of fatal "opioid" overdoses in the US also involve benzodiazepines, raising the possibility that some of the increase in opioid related deaths might be caused by increases in concurrent benzodiazepine/opioid use over time

Although the concurrent use of opioids and benzodiazepines has been studied among patients in the US Veterans Health Administration, trends in concurrent use among the privately insured population, and their consequences for population health, have not been fully characterized

\section{WHAT THIS STUDY ADDS.}

In a large sample of privately insured patients in 2001-13, concurrent

benzodiazepine/opioid prescribing increased by nearly $80 \%$

Opioid users who also used benzodiazepines were at substantially higher risk of an emergency room visit or inpatient admission for opioid overdose

Elimination of the concurrent use of benzodiazepines and opioids could reduce the population risk of an emergency room visit or inpatient admission for opioid

overdose by $15 \%$ room visit or inpatient admission for opioid overdose (adjusted odds ratio 2.14, 95\% confidence interval 2.05 to 2.24; $\mathrm{P}<0.001)$ among all opioid users. The adjusted odds ratio for an emergency room visit or inpatient admission for opioid overdose was 1.42 (1.33 to 1.51; $P<0.001)$ for intermittent opioid users and 1.81 (1.67 to 1.96; $\mathrm{P}<0.001)$ chronic opioid users. If this association is causal, elimination of concurrent benzodiazepine/opioid use could reduce the risk of emergency room visits related to opioid use and inpatient admissions for opioid overdose by an estimated $15 \%$ (95\% confidence interval 14 to 16 ).

\section{CONCLUSIONS}

From 2001 to 2013, concurrent benzodiazepine/opioid use sharply increased in a large sample of privately insured patients in the US and significantly contributed to the overall population risk of opioid overdose.

\section{Introduction}

In the US, the increased use of prescription opioids and the resulting potential for addiction and overdose impose substantial public burden of morbidity, mortality, and economic costs. ${ }^{12}$ Opioid prescriptions have increased sharply-nearly threefold-over the past fifteen years, ${ }^{3}$ with a concurrent increase in opioid related overdoses and deaths. ${ }^{345}$ As a result, policymakers and researchers have expended considerable effort towards finding ways to reduce the misuse of, and overdose from, opioids. ${ }^{6-15}$

Nearly $30 \%$ of fatal "opioid" overdoses also involve benzodiazepines, which are often used concurrently with opioids, ${ }^{16-18}$ raising the possibility that some of the increase in opioid related deaths could be caused by increases in concurrent benzodiazepine/opioid use over time. Although benzodiazepines have received less public safety attention than opioids, the combination of the two drugs is dangerous because benzodiazepines potentiate the respiratory depressant effects of opioids. ${ }^{19}$ Indeed, the US Food and Drug Administration (FDA) recently released a "black box" caution, warning patients and providers about the potential risks of combined use. Understanding the degree to which concurrent benzodiazepine/opioid use has increased over time, as well as the magnitude of its potential adverse effects, could have important implications for policy and clinical practice. These concerns are particularly salient in the US, but there is also some evidence of high rates of concurrent use internationally. For example, one study found that $47 \%$ of patients in methadone treatment programs in Spain also used benzodiazepines, ${ }^{20}$ while a another study reported that nearly $52 \%$ of Swiss patients in methadone treatment programs were "regular" benzodiazepine users. ${ }^{21}$ Studies have also found high rates of benzodiazepine use among heroin users in Australia. ${ }^{2223}$ 
A recent study examined the incidence of opioid and benzodiazepine use among the subset of the veteran population who receives care from the Veterans Health Administration (VHA). Nearly $30 \%$ of VHA patients who were prescribed opioids also received a concurrent prescription for benzodiazepines, defined as having at least one day's overlap between a benzodiazepine and opioid prescription in a given calendar year. ${ }^{2425}$ Moreover, this study found that co-prescribing was associated with a significantly higher risk of death than with the use of opioids alone. Similar results were found in studies examining opioid prescriptions in North Carolina $^{26}$ and in Ontario, Canada. ${ }^{27}$

As prescribing behaviors are likely to vary nationally and across clinical settings, ${ }^{2829}$ however, the applicability of these findings to the broader population (including to veterans, most of whom do not access VHA care) is unclear. For example, compared with the general population, veterans in the US have a higher prevalence of substance misuse and mental health disorders. ${ }^{30-32}$ We focused on concurrent benzodiazepine/opioid use in a privately insured population broadly representative of the entire US, in whom concurrent use was defined as one day of overlap in the time periods covered by each prescription. We have built on previous work by focusing on trends in concurrent benzodiazepine/opioid use over time and their effects on population health, which has not been fully characterized. Using a large dataset of administrative health claims data, we explored trends in concurrent use in 2001-13. In addition, we examined the degree to which patients using these two prescribed drugs have an increased risk of an emergency room visit or inpatient admission for opioid overdose. Finally, we examined the degree to which reducing concurrent use could reduce the risk of emergency room visits and inpatient admissions for opioid overdose at the population level.

\section{Methods}

Data

We obtained a sample of administrative health claims provided by Marketscan (Truven Health Analytics, Ann Arbor, MI). Marketscan provides patient level data on use and expenditures for the care of patients enrolled in private insurance plans through a participating employer, health plan, or government organization. The database has grown from six million beneficiaries to comprise over 35 million beneficiaries today. Compared with the general US population, the Marketscan population includes more women, is more likely to come from the southern areas of the US, and is less likely to be drawn from the western areas of the US. ${ }^{33}$ The data are frequently used in analyses of healthcare use and spending. ${ }^{34-37}$ Our data include all claims from 1 January 2001 to 31 December 2013, inclusive. As we used de-identified patient data, institutional review board approval was not required.

The information on inpatient and outpatient data claims provided details from specific encounters, including diagnosis codes (ICD-9 (international classification of diseases, ninth edition)), procedure codes (current procedural terminology, CPT), and date of service provision. For the pharmacy claims data, the information provided includes fill date, quantity supplied, and number of days supplied. The data also provide the National Drug Code, which can be linked to Red Book data (Truven Health Analytics, Ann Arbor, MI) to obtain the generic name and dose of the prescribed drug.

\section{Sample}

Our initial sample consisted of the 595410 patients who were continuously enrolled in a plan with medical and pharmaceutical benefits from 1 January 2001 to 31 December 2013. We restricted our analysis to patients who were continuously enrolled during the study period because, as noted above, the set of employers and health plans contributing data to Marketscan has markedly increased over time, leading to a large increase in the number of people in the database. Our approach thus reduces the risk of confounding that might occur because of changes over time in the underlying population reporting data to Marketscan.

From this sample, we identified and excluded patients with a history of cancer or those who received a diagnosis of cancer during the study period $(n=28780)$ as well as those aged under 18 or over 64 when they first entered the study ( $n=142789$ ), giving a sample of 423841 patients. Our final sample was the subset of patients (315428) who filled at least one prescription for an opioid during the study period. A flow diagram (fig A) describing the construction of our sample is in the appendix.

\section{Outcomes}

Our primary outcome was an emergency room visit or inpatient admission for opioid overdose within a given calendar year. Using methods described elsewhere, ${ }^{38}$ we defined opioid overdose to be an admission or visit with ICD-9 codes indicating either opioid related poisoning or a potential opioid related adverse event (such as respiratory depression) and an ICD-9 code corresponding to opioid overdose. For each opioid prescription, we defined a time interval starting the day the prescription was filled and lasting the number of days supplied in the prescription. We counted visits only if they occurred during this time interval or within seven days after the end of this interval. For example, if a patient received an opioid prescription on 1 January 2007 with 10 days' supply, we counted only visits that occurred between 1 January 2007 and 17 January 2007. In our sensitivity analyses we considered alternative definitions, such as visits occurring within 30 days of the time interval previously described.

\section{Variables}

Our key independent variable of interest was whether an opioid user also used a benzodiazepine concurrently within a given calendar year. First, we identified opioid use by isolating all prescriptions for outpatient opioids (table A in appendix), excluding prescriptions containing hydrocodone in a cough/cold formulation. We then isolated all prescriptions for a benzodiazepine (table B in appendix) and directly examined the degree of temporal overlap between prescriptions among individuals 
who filled a prescription for both classes of drugs. Specifically, for each opioid prescription, we defined an interval in which the prescription took effect as the interval starting on the day the prescription was filled and lasting up to the number of day's supply provided in the prescription. We defined a similar interval for a benzodiazepine prescription and quantified the total number of opioid prescription days that overlapped with a benzodiazepine prescription days. For example, suppose a given patient filled an opioid prescription and received a 30 day supply on 1 January 2001. If the same individual filled a benzodiazepine prescription on 20 January 2001 with 30 days' supply, then 11 out of the 30 days of the opioid prescription overlapped with a benzodiazepine prescription. For our baseline analyses, we defined concurrent use as having at least one day of overlap in a given calendar year, ${ }^{24} 39$ in line with previous studies. We also considered alternative definitions of concurrent opioid/baseline in our sensitivity analyses.

Our analysis included several controls for patients' demographics and health. Age and sex were directly obtained from the claims data. ICD-9 diagnosis codes were used to control for comorbidities including diabetes mellitus and congestive heart failure (table 1 provides a full list of comorbidities). ${ }^{40}$ For each comorbidity, we identified the earliest year with at least two claims containing the associated ICD-9 codes (table $B$ in appendix) and defined the patient as having a history of the given comorbidity from that year onwards. Finally, we also controlled for total healthcare spending in the time period before the first opioid prescription in a given year. To do so, we isolated all pharmacy, inpatient, and outpatient claims submitted before the earliest opioid prescription in a given year. We then summed the spending across all these claims and divided by the number of calendar days in the interval between 1 January of the given year and the date of the earliest opioid prescription.

\section{Analyses}

We first calculated the annual percentage of opioid users with concurrent benzodiazepine use. We stratified our analysis by intermittent and chronic opioid users. Following previous work, ${ }^{10}$ chronic users were defined as patients who filled more than 10 prescriptions or had more than 120 days' supply in a given year, with the remaining opioid users being defined as intermittent users. Because our study sample consisted of patients who were continuously enrolled during the study period, the average age of our population increased by one year annually. Therefore, we calculated age adjusted estimates using methods described in the appendix.

We then used multivariate logistic regression to estimate the association between concurrent benzodiazepine/opioid use and opioid overdose among opioid users. The dependent variable in this regression was an indicator variable that equaled 1 if the patient had at least one emergency room visit or admission for opioid overdose (using the methods described above) in the given calendar year and 0 otherwise. Our independent variable of interest was an indicator variable that equaled 1 if the opioid user met the criteria for concurrent benzodiazepine use in the given year and 0 otherwise. We also included controls for age, year, and the set of additional variables in table 1 .

Finally, we calculated the population attributable fraction (PAF) of concurrent benzodiazepine/opioid use to the risk of opioid overdose. This fraction represents the relative risk reduction for a given event at the population level under a counterfactual scenario for a specific risk factor. For example, the population attributable fraction has been used to describe the degree to which low birth weight would be reduced if maternal smoking could be eliminated entirely. ${ }^{41}$ In our case, we calculated the population level risk reduction that would occur if concurrent benzodiazepine/opioid use could be

\begin{tabular}{|c|c|c|c|c|}
\hline & No benzodiazepine $(n=53389)$ & With benzodiazepine $(n=5425)$ & $\begin{array}{l}\text { P value for } \\
\text { difference } \\
\text { between groups }\end{array}$ & $\begin{array}{l}\text { Hedge's } \mathrm{g} \text { for } \\
\text { standardized difference } \\
\text { between groups }\end{array}$ \\
\hline Men & $23194(43 ; 43$ to 44$)$ & $1888(35 ; 34$ to 36$)$ & $<0.001$ & -0.30 \\
\hline Mean age (years) ${ }^{*}$ & $42.4(42.4$ to 42.5$)$ & 44.5 (44.4 to 44.7$)$ & $<0.001$ & 0.18 \\
\hline Congestive heart failure & $79(0.15 ; 0.12$ to 0.18$)$ & $42(0.77 ; 0.54$ to 1.01$)$ & $<0.001$ & -0.14 \\
\hline Peripheral vascular disease & $81(0.15 ; 0.12$ to 0.18$)$ & $22(0.41 ; 0.24$ to 0.58$)$ & $<0.001$ & -0.06 \\
\hline Hypertension & $3686(6.9 ; 6.7$ to 7.1$)$ & $516(9.5 ; 8.7$ to 10$)$ & $<0.001$ & -0.10 \\
\hline Chronic obstructive pulmonary disease & $1041(2.0 ; 1.8$ to 2.1$)$ & $252(4.7 ; 4.1$ to 5.2$)$ & $<0.001$ & -0.19 \\
\hline Diabetes mellitus & $1813(3.4 ; 3.2$ to 3.6$)$ & $259(4.8 ; 4.2$ to 5.3$)$ & $<0.001$ & -0.07 \\
\hline Chronic kidney disease & $77(0.14 ; 0.11$ to 0.18$)$ & $12(0.22 ; 0.096$ to 0.35$)$ & 0.16 & -0.02 \\
\hline Cerebrovascular disease & $101(0.19 ; 0.15$ to 0.23$)$ & $35(0.65 ; 0.43$ to 0.86$)$ & $<0.001$ & -0.10 \\
\hline Dementia & $66(0.12 ; 0.09$ to 0.15$)$ & $15(0.28 ; 0.14$ to 0.42$)$ & $<0.001$ & -0.04 \\
\hline Myocardial infarction & $69(0.13 ; 0.10$ to 0.16$)$ & $22(0.41 ; 0.24$ to 0.58$)$ & $<0.001$ & -0.07 \\
\hline Liver disease & $251(0.47 ; 0.41$ to 0.53$)$ & $74(1.4 ; 1.1$ to 1.7$)$ & $<0.001$ & -0.12 \\
\hline Alcohol abuse & $162(0.30 ; 0.26$ to 0.35$)$ & $61(1.1 ; 0.84$ to 1.4$)$ & $<0.001$ & -0.13 \\
\hline Drug abuse & $118(0.22 ; 0.18$ to 0.26$)$ & $63(1.2 ; 0.88$ to 1.5$)$ & $<0.001$ & -0.17 \\
\hline Psychosis & $67(0.13 ; 0.10$ to 0.16$)$ & $30(0.55 ; 0.36$ to 0.75$)$ & $<0.001$ & -0.11 \\
\hline Depression & $2362(4.4 ; 4.3$ to 4.6$)$ & $915(17 ; 16$ to 18$)$ & $<0.001$ & -0.55 \\
\hline Mean daily health spending $(\$)^{\star}$ & $\$ 21.83$ (20.91 to 22.74) & $\$ 33.62$ (29.22 to 38.02$)$ & $<0.001$ & -0.10 \\
\hline
\end{tabular}


eliminated entirely. These estimates were calculated by using the results from the logistic analyses described above, following methods described elsewhere. ${ }^{4243}$

Because the unit of observation in our data is a person year, patients will contribute multiple observations if they used opioids in more than one calendar year. We therefore adjusted our standard errors for clustering at the patient level. ${ }^{44}$ All analyses were performed with Stata 14.0 (College Station, TX).

\section{Sensitivity analyses}

We conducted several sets of sensitivity analyses. First, in our baseline analyses we defined concurrent benzodiazepine/opioid overdose as requiring at least one day of overlap; we considered an alternative (stricter) definition that required $25 \%$ of the days' supply of opioids to overlap with a benzodiazepine prescription. Similarly, our baseline analyses defined an opioid overdose as an emergency room visit or admission occurring during the time interval covered by an opioid prescription or within seven days after the end of the prescription; we considered alternative definitions that both loosened (allowing a visit to occur within 30 days after the time interval covered by an opioid prescription) and tightened (requiring the visit to occur exactly during the interval covered by an opioid prescription) this criterion.

Second, a potential issue arises because our sample was constructed as a set of individuals who were continuously enrolled between 2001 and 2013. The advantage of this approach is that allows us to follow a uniform set of people over time. By contrast, other approaches-such as including all patients regardless of enrollment duration-would have the drawback of having to deal with a changing population over time as people enter and exit the sample. Restriction to people who did not leave the sample (because of death or loss of employment), however, could lead to bias because people who die (or lose employment) secondary to opioid use would probably have experienced a series of emergency room visits or inpatient admissions for opioid overdose before the actual event. To the degree that the concurrent benzodiazepine/opioid use increases the risk of these events (and of attrition), our approach will therefore underestimate the true effect of concurrent use (as our sample is limited to people who did experience these events, but not enough to result in death or loss of employment). To deal with this, we conducted a secondary analysis using a broader sample consisting of all people who were continuously enrolled for at least two years $(n=3810747)$. Each individual remained in the sample until the study end date or until they exited the sample. Thus, this broader sample includes our original sample as well as patients who subsequently entered and exited the sample.

Finally, one potential source of bias is that opioid users who concurrently used benzodiazepines could differ from those who did not. We performed a residual confounding analysis ${ }^{4546}$ to investigate the extent to which our results could be explained by other unobservable factors, such as differences in health status between the two groups. Specifically, we assumed the presence of an unmeasured binary confounder that was patient specific and independent of our measured confounders. We assumed that this confounder had a prevalence of $75 \%$ among our surgical sample and $0 \%$ among the non-surgical patients. The assumed difference in prevalence between surgical and non-surgical patients of this unmeasured confounder is much larger than the difference in prevalence for all the medical comorbidities we examined. Using methods described elsewhere, we then estimated the degree of confounding that would be necessary for this confounder to eliminate the estimated association between opioid overdose and concurrent benzodiazepine/opioid use. ${ }^{46}$

\section{Patient involvement}

No patients were involved in setting the research question or the outcome measures, nor were they involved in developing plans for design or implementation of the study. No patients were asked to advise on interpretation or writing up of results. There are no plans to disseminate the results of the research to study participants or the relevant patient community.

\section{Results}

Table 1 shows descriptive statistics for patients who filled at least one prescription for an opioid during the year, stratified by whether those patients also filled at least one prescription for a benzodiazepine. Among opioid users, patients who also filled a prescription for a benzodiazepine were older $(44.5 v 42.4, \mathrm{P}<0.001)$ and less likely to be men $(35 \% v 43 \%, \mathrm{P}<0.001)$ compared with those who did not. In addition, the prevalence of every comorbidity examined was significantly higher $(\mathrm{P}<0.001)$ among opioid users who also filled prescriptions for a benzodiazepine. Given our large sample size, we are likely to find many differences between the two groups that are small in magnitude but nonetheless significant. We therefore calculated the standardized difference between the two groups using Hedge's g (table 1). Overall, the differences between the two groups for most of the characteristics were of modest magnitude $(<0.2$ $\mathrm{SD}$ ), with the exception of depression (roughly 0.5 SD).

The proportion of opioid users who were co-prescribed benzodiazepines nearly doubled from 9\% (5425/53389) in 2001 to $17 \%$ (14415/85617) in 2013 (fig 1). Most of this growth occurred among intermittent opioid users, in whom the percentage of patients who also used a benzodiazepine increased from $7 \%(4122 / 55960)$ to $13 \%$ (9292/65114) during the study period. By contrast, although a higher percentage $(46 \% ; 1596 / 3457)$ of chronic opioid users also used benzodiazepines in 2001, this percentage remained fairly constant during the study period.

Among all opioid users who did not use benzodiazepines, the age adjusted incidence of emergency room visits or inpatient admissions for opioid overdose increased from 1.08\% (95\% confidence interval 0.99\% to $1.16 \%$ ) in 2001 to $1.35 \%$ (1.26\% to $1.43 \%$ ) from 2001 to 2013 (fig 2). For intermittent opioid users who did use not benzodiazepines, the age adjusted incidence of opioid overdose increased from $1.05 \%$ ( $0.96 \%$ to $1.14 \%)$ to $1.15 \%(1.06 \%$ to $1.23 \%)$ during this time period, and 
Fig $1 \mid$ Annual age adjusted prevalence of concurrent benzodiazepine/opioid use, 2001-13. Concurrent use was defined as having at least one day of overlap between time covered by prescriptions

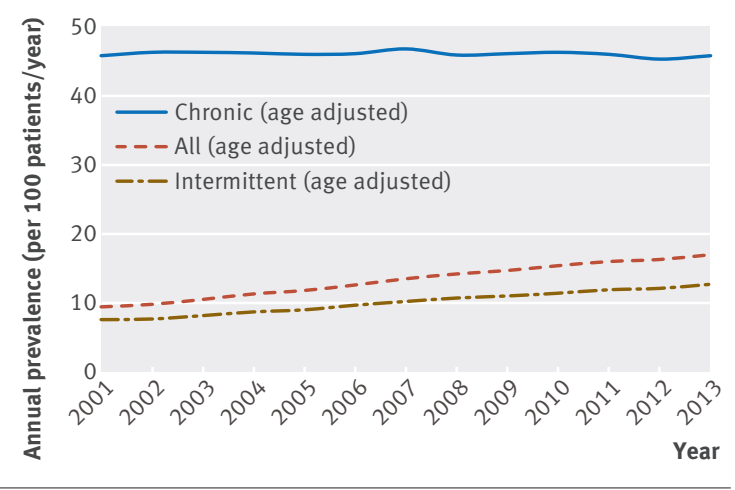

Fig 2 Unadjusted incidence of opioid overdose for patients using opioids with or without benzodiazepines in 2001-13 stratified by intermittent $v$ chronic opioid use and concurrent benzodiazepine/opioid use

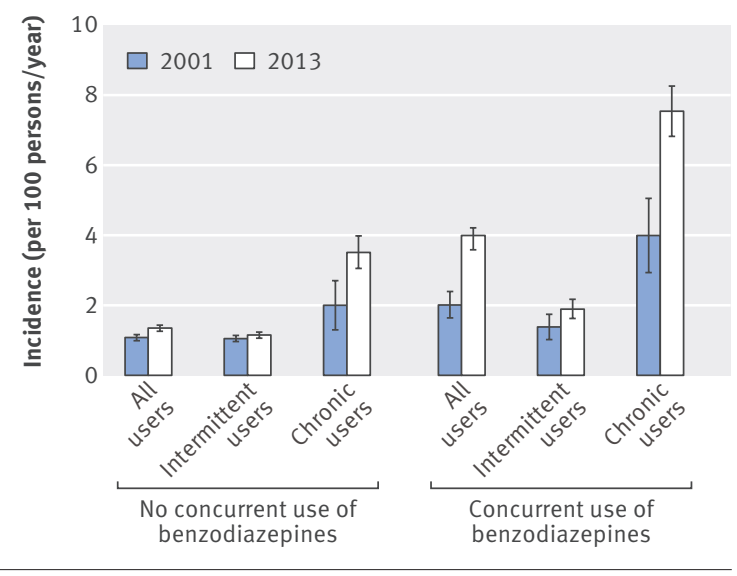

Fig 3 Adjusted incidence of opioid overdose for patients taking opioids with and without benzodiazepines. Adjusted incidence incorporates controls for year, sex, age, and characteristics listed in table 1 ( $95 \%$ confidence intervals calculated with SE clustered at patient level) admissions for opioid overdose $(1.45 \%, 1.36 \%$ to $1.51 \%)$ compared with intermittent opioid users who did not use a benzodiazepine concurrently $(1.02 \%, 0.996 \%$ to $1.04 \%$ ), with an odds ratio of 1.42 (1.33 to 1.51; $\mathrm{P}<0.001$ ). Chronic opioid users with concurrent benzodiazepine use also experienced a higher adjusted incidence of emergency room visits or inpatient admissions for opioid overdose $(5.36 \%, 5.12 \%$ to $5.61 \%)$ compared with those who did not use benzodiazepines (3.13\%, 2.94\% to $3.31 \%$ ), with an odds ratio of 1.81 (1.67 to 1.96 ; $\mathrm{P}<0.001$ ).

Using the logistic regression model results, we estimated the population attributable fraction for benzodiazepine co-prescribing to be 0.15 ( $95 \%$ confidence interval 0.14 to 0.16 ) among all opioid users, suggesting that eliminating concurrent benzodiazepine/opioid use could reduce the population risk for an opioid related emergency room visit or inpatient admission by $15 \%$. Among intermittent opioid users, the population attributable fraction was 0.043 (0.034 to 0.051 ), whereas the population attributable fraction was 0.27 (0.23 to 0.30 ) for chronic users.

We conducted three sets of sensitivity analyses. First, we considered alternative measures of concurrent benzodiazepine/opioid use (requiring $25 \%$ of the days of opioid to overlap with a benzodiazepine prescription) as well as alternative measures of opioid overdose. The results were qualitatively similar to our main results (appendix).

A second set of analyses examined whether imposing the requirement that our study population be continuously enrolled from 2001 to 2013 could have resulted in bias. This set of analyses used a broader sample, consisting of the patients in our original sample as well as patients who were continuously enrolled for at least two years but who might have subsequently left the sample. Among this larger sample, the adjusted relative risk was 1.66 for intermittent users (95\% confidence interval 1.64 to $1.69 ; \mathrm{P}<0.001)$ and 1.61 (1.58 to 1.63 ; $\mathrm{P}<0.001$ ) for chronic users.

Finally, we performed a residual confounding analysis to estimate the degree of confounding that would need to be present to explain our results. Assuming the presence of an unmeasured binary confounder with a prevalence of $75 \%$ among concurrent benzodiazepine/ opioid users or a prevalence of $0 \%$ among persons with no concurrent use, our analysis suggested that residual confounding would negate our results only if the odds ratio associated with the unmeasured confounder was at least 2.40. The odds ratio associated with this confounder would need to be at least 1.45 among intermittent users and at least 1.89 among chronic users.

\section{Discussion}

\section{Principal findings}

In a sample of privately insured patients, we found that the incidence of concurrent benzodiazepine/opioid use increased by roughly $80 \%$ from 2001 to 2013 . Moreover, we found that opioid users who concurrently used benzodiazepines were at an increased risk of opioid overdose and that eliminating concurrent benzodiazepine/opioid use could reduce the risk of opioid overdose by $15 \%$. Opioid 
prescribing, use, and overdose are receiving increased attention given the sharp increase in the number of opioid related adverse events over the past decade. Understanding the underlying causes for these secular increases in opioid related events is an important step towards developing policies aimed at reducing their incidence.

\section{Comparison with other studies}

A previous study of patients receiving care from the Veteran's Administration found that $27 \%$ of opioid users also received benzodiazepines and that concurrent opioid/benzodiazepine use was associated with an increased risk of death from opioid overdose. ${ }^{24}$ Another study found that nearly $80 \%$ of patients taking an opioid also used a benzodiazepine and that those who used both drugs concurrently were at a tenfold increased risk of death from overdose, ${ }^{26}$ although in that study concurrent use was defined as having used an opioid and benzodiazepine at least once in a given year, without an attempt to identify the extent of overlap between the periods of opioid and benzodiazepine use. Using toxicology analysis, another study found that benzodiazepines were involved in $60 \%$ of deaths from opioid overdose in patients in Ontario, Canada. ${ }^{27}$

We also found that concurrent opioid/benzodiazepine use was fairly common; differences in the magnitude of concurrent use between our studies and previous work is possibly because of differences in the definition of concurrent use as well as differences in the underlying patient population. Our study builds on these results by examining growth in concurrent use over time and estimating the effect of this growth on population health. Moreover, we examined concurrent use in a national sample that is broadly representative of the privately insured population in the US.

\section{Strengths and limitations of this study}

Our results should be viewed in the light of the study's limitations. First, we cannot exclude the possibility of confounding because of unobservable differences between opioid users who did and did not use benzodiazepines. While we dealt with this issue by adjusting for an extensive set of covariates and comorbidities, we cannot exclude the possibility of further confounding. We did, however, perform a residual confounding analysis to judge the extent of confounding that would be needed to explain our results. Our analysis suggested that any unobserved confounder would need to exert effects larger than the estimated effect for concurrent benzodiazepine/opioid use and be unequally distributed across concurrent and non-concurrent users to a far larger extent than any of the potential measured confounders we considered, a scenario we consider to be unlikely.

Second, the construction of our sample-which required people to be continuously enrolled for the entire 13 year period-could also result in confounding as it excluded those who might have left the sample secondary to opioid related death or job loss. To deal with this issue, we performed sensitivity analyses in which we added to our original sample individuals who were continuously enrolled for at least two years but who might have subsequently left the sample secondary to death or job loss. The point estimates for this set of sensitivity analyses were qualitatively similar to our baseline estimates.

Third, we note that our analysis examined only cases of opioid overdose/poisoning when a patient received emergency room/hospital care and ultimately survived, which could mean that our analysis underestimated the true risk of opioid overdose.

Fourth, our analysis does not take into account changes in prescribing/patient behaviors that could evolve in response to reduced concurrent benzodiazepine/opioid prescribing. For example, if patients increase their dose of opioids in response to a reduction in concurrent benzodiazepine/opioid prescribing, this would mitigate some of the benefits we observed in the study.

Finally, we note that a prescription database would not capture heroin use or the use of prescription drugs bought illegally.

\section{Conclusions and policy implications}

These findings have several implications. From a clinical perspective, providers should exercise caution in prescribing opioids for patients who are already using benzodiazepines (or vice versa), even in a non-chronic setting. Indeed, we note that the association between concurrent benzodiazepine/opioid use and the risk of opioid overdose was broadly similar for both intermittent and chronic opioid users. Therefore, opioids should be prescribed cautiously-even if only for a short term courseamong patients who are also using benzodiazepines. From a policy perspective, in addition to the current focus on opioid prescribing, policymakers and healthcare systems should also focus on benzodiazepine prescribing behaviors, as these behaviors can play an important role in mitigating the risks of opioid prescriptions. Healthcare systems might also want to implement education programs that warn prescribers and patients about the risks of taking benzodiazepines and opioids concurrently, with the Veterans Health Administration's system-wide opioid safety initiative being a potential model to emulate.

Contributors: All authors contributed to the design and conduct of the study, data collection and management, analysis interpretation of the data; and preparation, review, or approval of the manuscript. ECS is guarantor.

Funding: ECS was supported by a mentored research training grant from the Foundation for Anesthesia Education and Research and the Anesthesia Quality Institute. KNH was supported by funding from the Veterans Affairs health services research and development service. The research conducted was independent of any involvement from the sponsors of the study. Study sponsors were not involved in study design, data interpretation, writing, or the decision to submit the article for publication.

Competing interests: All authors have completed the ICMJE uniform disclosure form at www.icmje.org/coi_disclosure.pdf (available on request from the corresponding author) and declare: support provided by grants from the Foundation for Anesthesia Education and Research; no financial relationships with any organizations that might have an interest in the submitted work in the previous 3 years; and no other relationships or activities that could appear to have influenced the submitted work

Ethical approval: Not required as patient data were de-identified. Data sharing: No additional data available.

Transparency statement: The lead author affirms that the manuscript is an honest, accurate, and transparent account of the study being 
reported; that no important aspects of the study have been omitted; and that any discrepancies are disclosed.

This is an Open Access article distributed in accordance with the Creative Commons Attribution Non Commercial (CC BY-NC 4.0) license, which permits others to distribute, remix, adapt, build upon this work non-commercially, and license their derivative works on different terms, provided the original work is properly cited and the use is noncommercial. See: http://creativecommons.org/licenses/by-nc/4.0/.

1 Rudd RA, Aleshire N, Zibbell JE, Gladden RM. Increases in Drug and Opioid Overdose Deaths--United States, 2000-2014. MMWR Morb Mortal Wkly Rep 2016:64:1378-82. doi:10.15585/mmwr.mm6450a3.

2 Leider HL, Dhaliwal J, Davis EJ, Kulakodlu M, Buikema AR. Healthcare costs and nonadherence among chronic opioid users. Am J Manag Care 2011;17:32-40

3 Centers for Disease Control and Prevention (CDC). Vital signs: overdoses of prescription opioid pain relievers---United States, 1999--2008. MMWR Morb Mortal Wkly Rep 2011;60:1487-92.

4 Bohnert AS, Valenstein M, Bair MJ, et al. Association between opioid prescribing patterns and opioid overdose-related deaths. JAMA 2011;305:1315-21. doi:10.1001/jama.2011.370

5 Overdose Death Rates National Institute on Drug Abuse. http://www. drugabuse.gov/related-topics/trends-statistics/overdose-death-rates.

6 McDonald DC, Carlson K, Izrael D. Geographic variation in opioid prescribing in the U.S. J Pain 2012;13:988-96. doi:10.1016/j.jpain.2012.07.007.

7 McDonald DC, Carlson KE. The ecology of prescription opioid abuse in the USA: geographic variation in patients' use of multiple prescribers (“doctor shopping"). Pharmacoepidemiol Drug Saf 2014;23:1258-67. doi:10.1002/pds.3690.

8 Alam A, Gomes T, Zheng H, Mamdani MM, Juurlink DN, Bell CM. Long-term analgesic use after low-risk surgery: a retrospective cohort study. Arch Intern Med 2012:172:425-30. doi:10.1001/ archinternmed.2011.1827.

9 Clarke H, Soneji N, Ko DT, Yun L, Wijeysundera DN. Rates and risk factors for prolonged opioid use after major surgery: population based cohort study. BM/ 2014:348:g1251. doi:10.1136/bmj.g1251.

10 Raebel MA, Newcomer SR, Reifler LM, et al. Chronic use of opioid medications before and after bariatric surgery. JAMA 2013;310:136976. doi:10.1001/jama.2013.278344

11 Carroll I, Barelka P, Wang CK, et al. A pilot cohort study of the determinants of longitudinal opioid use after surgery. Anesth Analg 2012;115:694-702.

12 Paulozzi LJ, Kilbourne EM, Desai HA. Prescription drug monitoring programs and death rates from drug overdose. Pain Med 2011;12:74754. doi:10.1111/j.1526-4637.2011.01062.x

13 Friedly J, Nishio I, Bishop MJ, Maynard C. The relationship between repeated epidural steroid injections and subsequent opioid use and lumbar surgery. Arch Phys Med Rehabil 2008;89:1011-5. doi:10.1016/j.apmr.2007.10.037.

14 Sehgal N, Paidin M, Rasmussen D, Tarig A, Hetzel S. Is there a decrease in opioid use after a single epidural steroid injection in LBP: a pilot study. J Pain 2013;14(Supplement):S85doi:10.1016/j.jpain.2013.01.676.

15 Gugelmann HM, Perrone J. Can prescription drug monitoring programs help limit opioid abuse?JAMA 2011;306:2258-9. doi:10.1001/jama.2011.1712.

16 Morasco BJ, Duckart JP, Carr TP, Deyo RA, Dobscha SK. Clinical characteristics of veterans prescribed high doses of opioid medications for chronic non-cancer pain. Pain 2010;151:625-32. doi:10.1016/j.pain.2010.08.002.

17 Saunders KW, Von Korff M, Campbell Cl, et al. Concurrent use of alcohol and sedatives among persons prescribed chronic opioid therapy: prevalence and risk factors. J Pain 2012;13:266-75. doi:10.1016/j.jpain.2011.11.004.

18 Hermos JA, Young MM, Gagnon DR, Fiore LD. Characterizations of long-term oxycodone/acetaminophen prescriptions in veteran patients. Arch Intern Med 2004;164:2361-6. doi:10.1001/ archinte.164.21.2361.

19 Dowell D, Haegerich TM, Chou R. CDC Guideline for Prescribing Opioids for Chronic Pain--United States, 2016. JAMA 2016;315:162445. doi:10.1001/jama.2016.1464

20 Fernández Sobrino AM, Fernández Rodríguez V, López Castro [Benzodiazepine use in a sample of patients on a treatment program with opiate derivatives (PTDO)]. Adicciones 2009;21:143-6.

21 Meiler A, Mino A, Chatton A, Broers B. Benzodiazepine use in a methadone maintenance programme: patient characteristics and the physician's dilemma. Schweiz Arch Neurol Psychiatr 2005;156:310-7.

22 Darke S, Hall W, Ross M, Wodak A. Benzodiazepine use and HIV risk-taking behaviour among injecting drug users. Drug Alcohol Depend 1992;31:31-6. doi:10.1016/0376-8716(92)90005-W.

23 Ross J, Darke S. The nature of benzodiazepine dependence among heroin users in Sydney, Australia. Addiction 2000:95:1785-93. doi:10.1046/j.1360-0443.2000.951217858.x.
24 Park TW, Saitz R, Ganoczy D, Ilgen MA, Bohnert AS. Benzodiazepine prescribing patterns and deaths from drug overdose among US veterans receiving opioid analgesics: case-cohort study. BM 2015;350:h2698. doi:10.1136/bmj.h2698.

25 Jones JD, Mogali S, Comer SD. Polydrug abuse: a review of opioid and benzodiazepine combination use. Drug Alcohol Depend 2012;125:818. doi:10.1016/j.drugalcdep.2012.07.004.

26 Dasgupta N, Funk MJ, Proescholdbell S, Hirsch A, Ribisl KM, Marshall S. Cohort Study of the Impact of High-Dose Opioid Analgesics on Overdose Mortality. Pain Med 2016:17:85-98.

27 Gomes T, Mamdani MM, Dhalla IA, Paterson JM, Juurlink DN. Opioid dose and drug-related mortality in patients with nonmalignant pain. Arch Intern Med 2011:171:686-91. doi:10.1001/archinternmed.2011.117.

28 Paulozzi LJ, Mack KA, Hockenberry JM. Division of Unintentional Injury Prevention, National Center for Injury Prevention and Control, CDC. Vital signs: variation among States in prescribing of opioid pain relievers and benzodiazepines - United States, 2012. MMWR Morb Mortal Wkly Rep 2014;63:563-8.

29 Chen JH, Humphreys K, Shah NH, Lembke A. Distribution of Opioids by Different Types of Medicare Prescribers. JAMA Intern Med 2016;176:259-61. doi:10.1001/jamainternmed.2015.6662.

30 Vazan P, Golub A, Bennett AS. Substance use and other mental health disorders among veterans returning to the inner city: prevalence, correlates, and rates of unmet treatment need. Subst Use Misuse 2013;48:880-93. doi:10.3109/10826084.2013.796989.

31 Lan CW, Fiellin DA, Barry DT, et al. The epidemiology of substance use disorders in US Veterans: A systematic review and analysis of assessment methods. Am J Addict 2016;25:7-24. doi:10.1111/ajad.12319.

32 Wagner TH, Harris KM, Federman B, Dai L, Luna Y, Humphreys K. Prevalence of substance use disorders among veterans and comparable nonveterans from the National Survey on Drug Use and Health. Psychol Serv 2007;4:149-57doi:10.1037/1541-1559.4.3.149.

33 Aizcorbe A, Liebman E, Pack S, Cutler DM, Chernew ME, Rosen AB. Measuring health care costs of individuals with employer-sponsored health insurance in the U.S.: A comparison of survey and claims data. Stat) IAOS 2012;28:43-51.

34 Baker L, Bundorf MK, Royalty A. Private insurers' payments for routine physician office visits vary substantially across the United States. Health Aff(Millwood) 2013;32:1583-90. doi:10.1377/hlthaff.2013.0309.

35 Chernew ME, Sabik LM, Chandra A, Gibson TB, Newhouse JP. Geographic correlation between large-firm commercial spending and Medicare spending. Am J Manag Care 2010;16:131-8.

36 Dor A, Koroukian S, Xu F, Stulberg J, Delaney C, Cooper G. Pricing of surgeries for colon cancer: patient severity and market factors. Cancer 2012;118:5741-8. doi:10.1002/cncr.27573.

37 Dunn A, Liebman E, Pack S, Shapiro AH. Medical care price indexes for patients with employer-provided insurance: nationally representative estimates from MarketScan Data. Health Serv Res 2013;48:1173-90. doi:10.1111/1475-6773.12008

38 Dunn KM, Saunders KW, Rutter CM, et al. Opioid prescriptions for chronic pain and overdose: a cohort study. Ann Intern Med 2010;152:85-92. doi:10.7326/0003-4819-152-2-201001190-00006

39 Concurrent Use of Opioids and Benzodiazepines in a Medicare Part D Population. 2016. https://www.cms.gov/Medicare/PrescriptionDrug-Coverage/PrescriptionDrugCovContra/Downloads/ Concurrent-Use-of-Opioids-and-Benzodiazepines-in-a-MedicarePart-D-Population-CY-2015.pdf

40 Quan $\mathrm{H}$, Li B, Couris CM, et al. Updating and validating the Charlson comorbidity index and score for risk adjustment in hospital discharge abstracts using data from 6 countries. Am J Epidemiol 2011;173:67682. doi:10.1093/aje/kwq433.

41 Ojima T, Uehara R, Watanabe M, Tajimi M, Oki I, Nakamura Y. Population attributable fraction of smoking to low birth weight in Japan. Pediatr Int 2004;46:264-7. doi:10.1111/i.1442-200x.2004.01881.x

42 Brady A. Adjusted population attributable fractions from logistic regression.Stata Tech Bull 1998;42:8-12.

43 Greenland S, Drescher K. Maximum likelihood estimation of the attributable fraction from logistic models. Biometrics 1993:49:865-72. doi:10.2307/2532206.

44 Bertrand M, Duflo E, Mullainathan S. How Much Should We Trust Differences-in-Differences Estimates? Q I Econ 2004;119:249-75doi:10.1162/003355304772839588.

45 Karaca-Mandic P, Jena AB, Joyce GF, Goldman DP. Out-of-pocket medication costs and use of medications and health care services among children with asthma. JAMA 2012;307:1284-91. doi:10.1001/ jama.2012.340.

46 Lin DY, Psaty BM, Kronmal RA. Assessing the sensitivity of regression results to unmeasured confounders in observational studies. Biometrics 1998;54:948-63. doi:10.2307/2533848.

Appendix: Supplementary content 